\title{
Self-pulsing laser as oscillator Toda: approximations through elementary functions
}

\author{
D Kouznetsov, J-F Bisson, J Li and K Ueda \\ Institute for Laser Science, University of Electro-Communications, 1-5-1 Chofu, Tokyo, \\ 182-8585, Japan \\ E-mail: dima@ils.uec.ac.jp
}

Received 20 July 2006, in final form 15 January 2007

Published 14 February 2007

Online at stacks.iop.org/JPhysA/40/2107

\begin{abstract}
A simple model of self-pulsation in lasers is considered. The laser is described by the system of two ordinary differential equations for the number of photons in the cavity and the number of excitations in the active medium, leading to the equation for the oscillator Toda with damping. For the case of strong spiking, the damping is considered as perturbation; the estimates in terms of elementary functions are suggested for the period of pulsation, damping rate, amplitude and phase of pulsation, quasi-energy and the output power. These estimates are compared to the numerical solution and to the experimental data.
\end{abstract}

PACS numbers: 42.60.Rn, 02.30.Hq, 02.30.Mv

(Some figures in this article are in colour only in the electronic version)

\section{Introduction}

Self-pulsation takes place at the beginning of laser action in various optical generators. As the pump is switched on, the gain in the active medium rises and exceeds the steady-state value. Then the number of photons in the cavity increases, depleting the gain below the steady-state value, and so on. The laser pulsates; the output power at the peaks can be orders of magnitude larger than that between pulses. After several strong peaks, the amplitude of pulsation reduces, and the system behaves as a linear oscillator with damping. Then the pulsation decays; this is the beginning of the continuous-wave operation [1-3]. The self-pulsation is a transient phenomenon in the continuous-wave lasers.

This paper was motivated by questions about applicability of analytical estimates of the efficiency of the laser action in various models $[4,5]$. The condition of pumping above the threshold and the sufficient cooling may require the quasi-continuous operation. With pulsed pump, the transient regime takes place at the beginning of each pulse. 
The pulsation in lasers has been under intensive study [6-29]. Usually, the analysis of pulsation is limited by the cases of a weak pulsation and/or the numerical solution of the differential equations, but the analytical properties of the solution can be revealed, treating the equations as oscillator Toda $[3,28,29]$ in the first-order averaging for the slow damping. Such a description makes it easier to understand the phenomenon. However, the use of these results implies the numerical solution of the differential equation for the quasi-energy of the oscillator Toda, while the right-hand side of this equation is expressed in terms of an integral. Therefore, numerically, such an 'analytic solution' does not run faster than a direct implementation of the second-order differential equation for the same oscillator.

The detailed description of any phenomenon should be followed by the easy-to-use portable algorithm which allows us to estimate physical quantities without repeating the calculus which is already done. In this paper, we suggest approximations for the main parameters of self-pulsation. Our goal is to describe the pulsation through simple functions, satisfying criteria $1-3$ below:

(1) Smallness of damping (necessary condition for the strong spiking) should be the only restriction for the validity of the approximation; other parameters may vary many orders of magnitude, covering all the physical range. (In particular, the approximation should reproduce the properties of the solution at very weak and at very strong pulsing.)

(2) The approximations should be expressed in terms of elementary functions; all the range of parameters should be covered with the same closed-form expressions. (For example, a step-function should not be used for the representation.)

(3) The error of the approximations for the model should be small compared to the deviation of the model from the experimental data.

In this paper, the evolution of the number of photons in the cavity and number of excitations is described by a system of two differential equations [1] (section 2). This system leads to the equation for the logarithm of the number of photons (section 3), which can be interpreted as oscillator Toda [3]. It is a nonlinear classical oscillator with a fixed (parameter independent) potential, and the damping term depends on two parameters.

The case without damping is considered in section 4. The period of pulsation is approximated through elementary functions with seven significant figures. The approximations are suggested for the number of photons as a function of time; the last one provides at least three correct significant figures.

The damping is considered as perturbation. In the first order averaging, the two parameters of damping are equivalent [3], and the scaling of the time variable allows us to describe the amplitude (section 5) and phase (section 6) of pulsation with fixed (parameter-independent) functions of single variable. These functions are approximated with three significant figures.

As an additional check and illustration, the resulting approximation for the output power versus time is compared with the numerical solution (section 7) and to the experimental realization (section 8 ) of the oscillator Toda.

\section{Model and notations}

In the simple model $[1,2]$ of self-pulsation, the evolution of the number $X$ of photons in the cavity and number $Y$ of the excitation of the active medium is described with the differential equations

$$
\begin{aligned}
& \mathrm{d} X / \mathrm{d} t=K X Y-U X \\
& \mathrm{~d} Y / \mathrm{d} t=-K X Y-V Y+W
\end{aligned}
$$


where

$$
\begin{aligned}
& K=\sigma /\left(s t_{\mathrm{r}}\right), \\
& U=\theta / L \\
& V=1 / \tau, \\
& W=P_{\mathrm{p}} /\left(\hbar \omega_{\mathrm{p}}\right)
\end{aligned}
$$

$t_{\mathrm{r}}$ is the round-trip time of light in the laser resonator, $s$ is area of the pumped region (good mode matching is assumed), $\sigma$ is the emission cross-section at the signal frequency $\omega_{\mathrm{s}}, \theta$ is the transmission coefficient of the output coupler, $\tau$ is the lifetime of excitation of the active medium and $P_{\mathrm{p}}$ is power of pump absorbed in the active medium.

Let $\omega_{\mathrm{p}}=2 \pi c / \lambda_{\mathrm{p}}$ and $\omega_{\mathrm{s}}=2 \pi c / \lambda_{\mathrm{s}}$ be frequencies of the pump and that of the lasing, then $\lambda_{\mathrm{p}}$ and $\lambda_{\mathrm{s}}$ are the corresponding wavelengths; the output power

$$
P_{\text {out }}=\theta \hbar \omega_{\mathrm{s}} X / t_{\mathrm{r}} \text {. }
$$

Both numbers $X$ and $Y$ are supposed to be large compared to unity; only in this case they can be treated as classical (commutative and real) continuous variables. System of equations (1), (2) is equivalent to the system (25.1), (25.2) in [1]. The steady-state solution

$$
X_{\mathrm{o}}=\frac{W}{U}-\frac{V}{K}, \quad Y_{\mathrm{o}}=\frac{U}{K}
$$

corresponds to the continuous-wave operation of the laser. It is pumped above threshold at $K W>U V$. In the case of weak pulsation $\left(\left|X-X_{\mathrm{o}}\right| \ll X_{\mathrm{o}}\right)$, the first term of the expansion of the solution in vicinity of $\left\{X_{0}, Y_{0}\right\}$ gives the estimate $[1,2]$ of the decay rate $\Gamma$ and frequency $\Omega$ of pulsation:

$$
\Gamma=K W /(2 U), \quad \Omega=\sqrt{w^{2}-\Gamma^{2}},
$$

where

$$
w=\sqrt{K W-U V} .
$$

The pulsation is possible at $w>\Gamma$. At the low damping, $\Gamma / w \ll 1$, and the frequency $\Omega$ of weak pulsation becomes $w$, but the repetition rate of strong pulses depends on the amplitude.

\section{Dimensionless variables}

The solution becomes simpler in new variables $x, y, z$ instead of $X, Y$, $t$; let

$$
\begin{aligned}
& X=X_{\mathrm{o}} e^{x}, \\
& Y=Y_{\mathrm{o}}+X_{\mathrm{o}} y, \\
& t=z / w .
\end{aligned}
$$

Then, the system (1), (2) leads to the equation

$$
\frac{\mathrm{d}^{2} x}{\mathrm{~d} z^{2}}+D(x) \frac{\mathrm{d} x}{\mathrm{~d} z}+\Phi^{\prime}(x)=0,
$$

where

$D(x)=u e^{x}+v, \quad u=w / U, \quad v=V / w, \quad \Phi(x)=e^{x}-x-1$, 


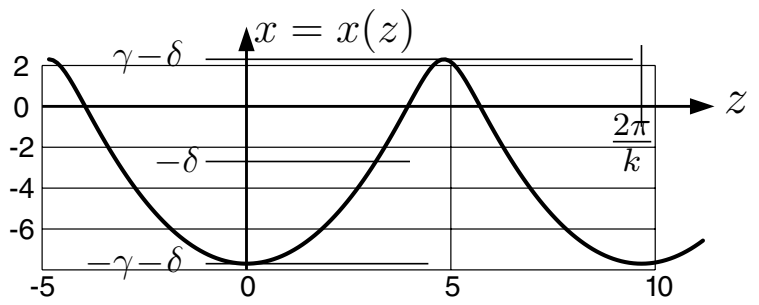

Figure 1. Solution of equation (16) with $\gamma=5$ and notations.

and prime denotes the derivative. Equation (14) can be interpreted as oscillator Toda [3], and treated as any other classical oscillator [30-32]. The independent variable $z$ is proportional to time $t$ by (27); variable $x$ appears as a coordinate of this oscillator; then, $\Phi(x)$ is the potential. The $\mathrm{d} x$ /dz plays the role of the speed of this particle; and the term with first derivative in (14) describes the speed-proportional friction; the coefficient of proportionality $D(x)$ is increasing function of coordinate $x$. The two functions $D$ and $\Phi$ specify the nonlinearity of the oscillator. Through $D(x)$, this nonlinearity is determined by two parameters $u$ and $v$. The shape of potential $\Phi$ does not depend on any parameters; it is fixed function.

\section{Case without damping}

For the strong spiking, both parameters $u$ and $v$ should be small; then, during one cycle of pulsation, the relaxation term can be treated as perturbation. Formally, we should also require something like $|\mathrm{d} x / \mathrm{d} z| u \ll 1$, but, in the physical range of parameters, maximal value of $|\mathrm{d} x / \mathrm{d} z|$ remains of order of unity even at strong spiking. Therefore, practically, the condition $u+v \ll 1$ is sufficient.

Let the 'unperturbed' equation correspond to the oscillator without damping:

$$
\frac{\mathrm{d}^{2} x}{\mathrm{~d} z^{2}}+\Phi^{\prime}(x)=0
$$

This equation has periodic solutions: one of them is plotted in figure 1 to show the notations. The energy of such oscillator can be expressed as follows:

$$
E=\frac{1}{2}\left(\frac{\mathrm{d} x}{\mathrm{~d} z}\right)^{2}+\Phi(x) .
$$

This dimensionless energy should not be confused with the energy of pulses

$$
E_{\text {pulse }}=\frac{\theta \hbar \omega_{\mathrm{s}}}{t_{\mathrm{o}}} X_{\mathrm{o}} \int_{0}^{2 \pi /(w k)} \exp (x(w t)) \mathrm{d} t,
$$

and the optical frequency $\omega_{\mathrm{s}}$ ('omega') should not be confused with the frequency $w$ ('lowercase double-u') of small pulsations; so, below $E$ is called 'quasi-energy', and the normalized frequency of pulsations is denoted by $k$; at weak pulsation, $k \approx 1$.

Neglecting the damping term leads to the conservation of quasi-energy, and the analytic solution of (16) during one period can be written as

$$
z= \pm \int_{x}^{x_{\max }} \frac{\mathrm{d} a}{\sqrt{2} \sqrt{E-\Phi(a)}}
$$

where

$$
E=\Phi\left(x_{\max }\right)=\Phi\left(x_{\min }\right)
$$


$x_{\min }$ and $x_{\max }$ are minimal and maximal values of $x$, which correspond to $\mathrm{d} x / \mathrm{d} z=0$. Equation (20), through the definition (15) of $\Phi$, leads to the relation between maximal and minimal values of $x$,

$$
E+1=\exp \left(x_{\min }\right)-x_{\min }=\exp \left(x_{\max }\right)-x_{\max }
$$

Let $X_{\max }=X_{\mathrm{o}} \exp \left(x_{\max }\right)$ and $X_{\min }=X_{\mathrm{o}} \exp \left(x_{\min }\right)$ be maximum and minimum value of the number of photons in the cavity. Define the amplitude of pulsation as $X_{\max } / X_{\min }-1$. Define the logamplitude

$$
\gamma=\frac{1}{2}\left(x_{\max }-x_{\min }\right)=\frac{1}{2} \ln \left(X_{\max } / X_{\min }\right)
$$

of pulsation and the median value

$$
-\delta=\frac{1}{2}\left(x_{\max }+x_{\min }\right)
$$

Then

$$
x_{\max }=-\delta+\gamma, \quad x_{\min }=-\delta-\gamma
$$

and (21) gives

$$
\begin{aligned}
& \delta=\delta(\gamma)=\ln \frac{\sinh (\gamma)}{\gamma} \\
& E=E(\gamma)=\frac{\gamma}{\tanh (\gamma)}+\ln \frac{\sinh \gamma}{\gamma}-1
\end{aligned}
$$

Any of extremal values $X_{\min }$ or $X_{\max }$, or $x_{\min }$, or $x_{\max }$, as well as quasi-energy $E$ or logamplitude $\gamma$ could be used to characterize the strength of pulsation. It is better to use logamplitude $\gamma$; then $x_{\min }, x_{\min }$ and $E$ are expressed through elementary functions of $\gamma$. The use of logamplitude $\gamma$ as parameter simplifies the deduction.

The time interval between pulses can be estimated as

$$
T(\gamma)=\frac{2 \pi}{w k(\gamma)}=\frac{2 \pi}{k(\gamma) \sqrt{W K-U V}}
$$

where the normalized frequency

$$
k(\gamma)=\pi \sqrt{2}\left(\int_{-\delta-\gamma}^{-\delta+\gamma} \frac{\mathrm{d} a}{\sqrt{e^{-\delta-\gamma}+\delta+\gamma+a-e^{a}}}\right)^{-1} .
$$

The normalized period $w T=2 \pi / k(\gamma)$ is shown in figure 2. The expansion of normalized period at small values of $\gamma$ can be written as follows:

$$
\frac{2 \pi}{k(\gamma)}=1-\sum_{n=1}^{\infty} T_{n}\left(-\gamma^{2}\right)^{n}
$$




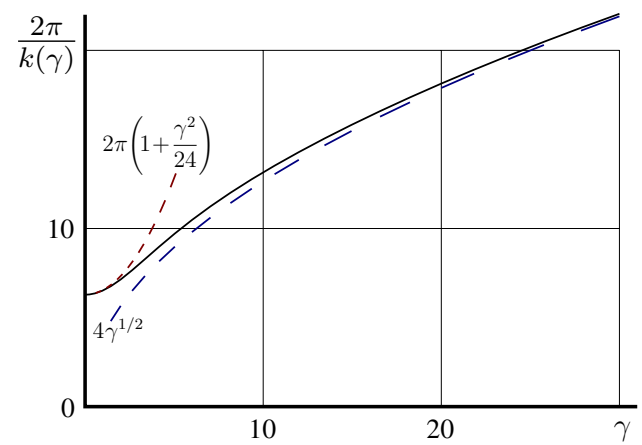

Figure 2. Normalized period of pulsation (solid curve) versus logamplitude $\gamma$ and two its asymptotic (dashed).

the first coefficients of the expansion are

$T_{1}=$

$T_{2}=$

$T_{3}=$

$T_{4}=$

$T_{5}=$

$T_{6}=$

$T_{7}=$

$T_{8}=$

$T_{9}=$

$T_{10}=$ $\frac{1}{24}$

$\frac{13}{6912}$

253

2488320

$$
\frac{61339}{33443020800}
$$$$
\frac{723077}{802632499200}
$$

$$
14228449243
$$

800963023601664000

62148265493

$\frac{62148265493}{11900022064939008000}$

199627352068763

$\frac{199627352068763}{5757706675900089630720000}$

7122419674276685557

$\frac{7122419674276685557}{739980461986679519340134400000}$

67823774202226535985551

67823774202226535985551

These coefficients can be obtained by integrating the expansion of the integrand of the representation

$\frac{\pi}{k(\gamma)}=\sqrt{\gamma \sinh (\gamma)} \int_{0}^{\pi} \frac{\sin (\phi) \mathrm{d} \phi}{\sqrt{[1-\cos (\phi)] \sinh (\gamma)+\exp (-\gamma)-\exp (-\gamma \cos \phi)}}$.

The expansion (29) can be used for precise evaluation of $k(\gamma)$ at $\gamma<1$; each coefficient in (30) is an order of magnitude smaller than the previous one.

At $\gamma \gg 1$, the quarter-period $\pi /(2 k)$ can be approximated with

$$
\frac{\pi}{2 k(\gamma) \sqrt{\gamma}} \approx 1+\sum_{n=1}^{N-1} K_{n} / \gamma^{n}+\mathcal{O}\left(1 / \gamma^{N}\right) ;
$$

the coefficients

$K_{1} \approx 0.346574, K_{2} \approx 0.2160, K_{3} \approx 0.199, K_{4} \approx 0.27, K_{5} \approx 0.5, K_{6} \approx 1.2$

were estimated from the numerical analysis of the integral (31). The left-hand side of (32) was evaluated using expression (31) at $10<\gamma<1000$ and approximated with $1-K_{1} \alpha$ at small values of $\alpha=1 / \gamma$. Then, the residual was divided by $\alpha$ and approximated as $K_{2} \alpha$, and so on. The accumulation of errors at the sequential evaluations leads to the quick drop of the number of significant figures; the last digit in coefficients (33) may be doubtful. The series seems to be strongly divergent; the more terms are taken into account in the sum in (32), the narrower 


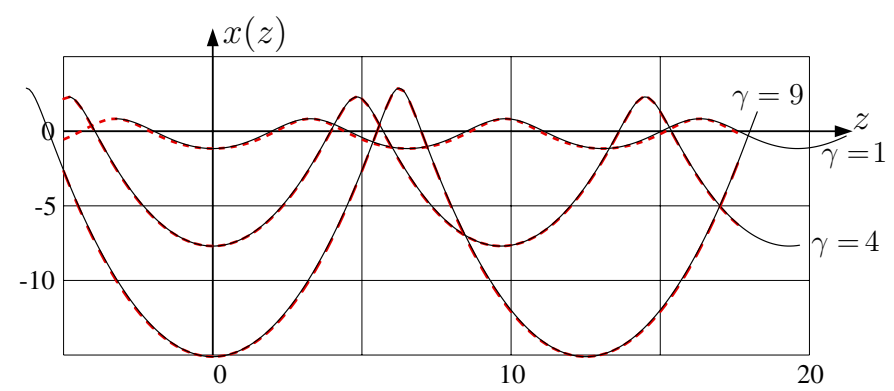

Figure 3. Evolution of $x(z)$ neglecting the damping of pulsation: approximation $x_{1}$ for $\gamma=1, \gamma=4$ and $\gamma=9$ (dashed) compared to the numerical solution (solid).

is range of validity of the resulting approximation. In particular, at $N=4$, this representation allows the precise evaluation of $k(\gamma)$ at $\gamma>100$, giving at least 8 significant figures. Such precision is important for the accurate estimates of behaviour of the amplitude and phase of pulsation at very strong spiking, described in the following sections.

However, at $\gamma=100$, the ratio $X_{\max } / X_{\min }=\mathrm{e}^{2 \gamma} \approx 10^{87}$; this greatly exceeds the physical range, at least for lasers. If we have only one photon in the gap between pulses, and the energy of a photon is of order of one electron Volt, then, the energy, stored in the cavity, should be of order of $10^{87} \times 10^{-19} \mathrm{~J}=10^{68} \mathrm{~J}$. This would correspond to an explosion of a heavy star rather than to an Earth-based laser. Values $0<\gamma<20$ may have physical meaning; the corresponding $x_{\max }<\ln (2 \gamma)<4$. We may expect, at any realization of the oscillator Toda as a self-pulsing laser, values of $x$ do not exceed the upper grid-line in figure 3. This is justification why we write the condition of small damping simply as $u+v \ll 1$, without specifying $x$.

Aiming application to the laser science, it would be good to have an easy estimate, valid at the intermediate values of logamplitude $\gamma$. In the whole range $\gamma>0$, the normalized frequency can be approximated with

$k_{\text {fit }}(\gamma)$
$=\left(\frac{10630+674 \gamma+695.2419 \gamma^{2}+191.4489 \gamma^{3}+16.86221 \gamma^{4}+4.082607 \gamma^{5}+\gamma^{6}}{10630+674 \gamma+2467 \gamma^{2}+303.2428 \gamma^{3}+164.6842 \gamma^{4}+36.6434 \gamma^{5}+3.9596 \gamma^{6}+0.8983 \gamma^{7}+\frac{16}{\pi 4} \gamma^{8}}\right)^{1 / 4}$

with eight significant figures; $\left|k_{\mathrm{fit}}(\gamma) / k(\gamma)-1\right|<22 \times 10^{-9}$ for all positive $\gamma$.

With the good approximation for the frequency of pulsation, the evolution of coordinate $x$ can be approximated with an appropriate periodic function. Let

$\beta(\gamma)=\sqrt{\frac{\gamma}{1-e^{-2 \gamma}}}, \quad c(\varphi)=1-\cos (\varphi)$,

$x_{0}(\gamma, \varphi)=-\delta-\gamma \cos (\varphi)$,

$x_{1}(\gamma, \varphi)=-\delta+\gamma-2 \ln \left(\cosh \left(\frac{\beta}{k} \sqrt{2 c(\varphi)}\right)\right)+c(\varphi)\left(\ln \left(\cosh \frac{2 \beta}{k}\right)-\gamma\right)$,

$x_{2}(\gamma, \varphi)=\frac{1}{1+\gamma^{2}} x_{0}(\gamma, \varphi)+\frac{\gamma^{2}}{1+\gamma^{2}} x_{1}(\gamma, \varphi)$.

The last argument $\varphi$ of the functions $x_{j}$ has sense of phase of pulsation. Then, the evolution can be approximated with $x(z)=x_{j}(\gamma, k(\gamma) z)$ for $j=0$ or 1 or 2 . 
The cosinusoidal approximation with $x_{0}$ gives relative error smaller than $1 \%$ at $\gamma \leqslant 1$; while the oscillation of $x$ is almost harmonic. The approximation with $x_{1}$ does well at $x \geqslant 4$; this fit is compared to the numerical solution in figure 3 , the deviation becomes visible at $\gamma \approx 1$. The combination $x_{2}$ coincides with the numerical solution with three decimal digits for all positive values of $\gamma$; the deviation would not be seen at the same graphic. The error of this approximation is small compared with the deviation from experimental data of the low-damping pulsation in a microchip laser discussed below.

The approximations through elementary functions of normalized frequency $k$ of pulsation and $x(z)$ can be used also for the case with small damping. However, the rate of decay of logamplitude $\gamma$ should be approximated.

\section{Damping as perturbation}

In the following, $u$ and $v$ by (15) are treated as small parameters; the relaxation term with first derivative in (14) appears as a perturbation. This does not assume small amplitude of pulsation, but the relative variation of the number of photons from pulse to pulse should not be large. The necessary condition for the strong pulsation is $u \ll 1, v \ll 1$. Their product also should be small: the definitions (15) give

$$
u v=V / U \ll 1 \text {. }
$$

In such a way, for the strong pulsation with low damping, the lifetime of photons in the cavity should be small compared to the lifetime of excitations of the active medium.

At the low damping, the quasi-energy $E$ and the logamplitude $\gamma$ of pulsation reduce slowly. Through $\gamma$, the extremal values of $x$ also become slow functions of the normalized coordinate $z$. The loss of quasi-energy during half a period $\pi / k$ can be estimated as

$$
\mathcal{E}=\int_{z_{\min }}^{z_{\max }} \mathrm{d} z\left(u e^{x}+v\right)\left(\frac{\mathrm{d} x}{\mathrm{~d} z}\right)^{2},
$$

where $z_{\min }$ and $z_{\max }$ are neighbour values of normalized coordinate $z$, at which variable $x$ (and number of photons $X$ ) have minimal and maximal values:

$$
x\left(z_{\min }\right)=x_{\min }, \quad x\left(z_{\max }\right)=x_{\max } .
$$

For the first order approximation with respect to $u$ and $v$, the derivative $\frac{\mathrm{d} x}{\mathrm{~d} z}$ can be expressed from (17). With $x$ as new variable of integration, the loss per half-period can be considered as a function of $\gamma$ :

$$
\mathcal{E}(\gamma)=\sqrt{2} \int_{-\gamma-\delta}^{\gamma-\delta}\left(u e^{x}+v\right) \sqrt{E(\gamma)-\Phi(x)} \mathrm{d} x,
$$

where $\delta$ and $E$ are determined with (25) and (26).

The smoothed evolution of quasi-energy can be estimated with

$$
\frac{\mathrm{d} E}{\mathrm{~d} z}=-\frac{\mathcal{E}}{\pi / k} \text {. }
$$

Considering $\gamma$ as parameter which determines the quasi-energy, (43) gives the equation for the logamplitude $\gamma$ :

$$
E^{\prime}(\gamma) \frac{\mathrm{d} \gamma}{\mathrm{d} z}=-\frac{k(\gamma)}{\pi} \mathcal{E}(\gamma)
$$

where $E^{\prime}$ is derivative of function $E$ by (26). Using (42), equation (44) can be rewritten as

$$
\frac{\mathrm{d} \gamma}{\mathrm{d} z}=-\frac{k(\gamma)}{E^{\prime}(\gamma)}(A(\gamma) u+B(\gamma) v)
$$


where

$$
\begin{aligned}
& A(\gamma)=\sqrt{2} \int_{-\gamma-\delta}^{\gamma-\delta} e^{x} \sqrt{E(\gamma)-\Phi(x)} \mathrm{d} x, \\
& B(\gamma)=\sqrt{2} \int_{-\gamma-\delta}^{\gamma-\delta} \sqrt{E(\gamma)-\Phi(x)} \mathrm{d} x .
\end{aligned}
$$

It is possible to show [3] that $A(\gamma)=B(\gamma)$; in the first approximation, parameters $u$ and $v$ give additive contribution to the damping, even at strong pulsation. These functions can be approximated with series of $\gamma$ :

$$
A(\gamma)=B(\gamma)=\frac{\pi \gamma^{2} / 2}{1-\sum_{n=1}^{\infty} a_{n}\left(-\gamma^{2}\right)^{n}}
$$

the first coefficients of the expansion

$$
\begin{aligned}
& a_{1}=\quad \frac{5}{144} \quad \approx 3.472222222222222 \times 10^{-2} \\
& a_{2}=\quad \frac{1}{640} \quad=1.5625 \times 10^{-3} \\
& a_{3}=\quad \frac{98069}{1045094400} \\
& \approx 9.383745621448168 \times 10^{-5} \\
& a_{4}=\quad \frac{1854883}{300987187200} \\
& a_{5}=\quad \frac{2388528323}{5562243219456000} \\
& \approx 6.162664322210723 \times 10^{-6} \\
& a_{6}=\frac{4926525386647}{156187789602324480000} \\
& a_{7}=\frac{218666888547727}{89964166810938900480000} \\
& \approx 4.294181733451065 \times 10^{-7} \\
& \approx 3.154232094064849 \times 10^{-8} \\
& \approx 2.430599829899596 \times 10^{-9} \\
& a_{8}=\frac{44570765432050679}{228389031477370222018560000} \\
& \approx 1.951528282410836 \times 10^{-10}
\end{aligned}
$$

can be obtained in the similar way, as the expansion (29), (30). The expansion (48), (49) allows the precise evaluation of $A(\gamma)$ at $\gamma<1$.

In the opposite case of large $\gamma$, the expansion seems to have the form

$$
A(\gamma) \approx \frac{8 \gamma^{3 / 2}}{3}\left[1-\sum_{n=1}^{N-1} A_{n} / \gamma^{n}+\mathcal{O}\left(\gamma^{-N}\right)\right]
$$

the first coefficients of the expansion

$$
A_{1} \approx 0.460279, \quad A_{2} \approx 0.128, \quad A_{3} \approx 0.09
$$

are estimated from the numerical analysis of expressions (46) and (47) at $10<\gamma<1000$ in the similar way as coefficients $K$ by (33). At $\gamma>100$, this expansion is suitable for the evaluation of function $A$. Such large values are out of the physical range, but they helped to build up the approximations below.

For $\gamma>0, A(\gamma)$ can be approximated by

$$
\begin{aligned}
& A_{\text {fit }}(\gamma) \\
& \quad=\frac{\pi \gamma^{2}}{2}\left(\frac{580+82 \gamma+33.8822 \gamma^{2}+8.20989 \gamma^{3}+\gamma^{4}}{580+82 \gamma+114.4 \gamma^{2}+19.6798 \gamma^{3}+6.23603 \gamma^{4}+1.2099 \gamma^{5}+(3 \pi / 16)^{4} \gamma^{6}}\right)^{1 / 4}
\end{aligned}
$$

with at least six significant figures, $\left|A_{\text {fit }}(\gamma) / A(\gamma)-1\right|<10^{-6}$.

The equality $A(\gamma)=B(\gamma)$ simplifies (45). Consider the new variable

$$
\zeta=\frac{z}{u+v}=\frac{\left(t-t_{1}\right) w}{u+v},
$$



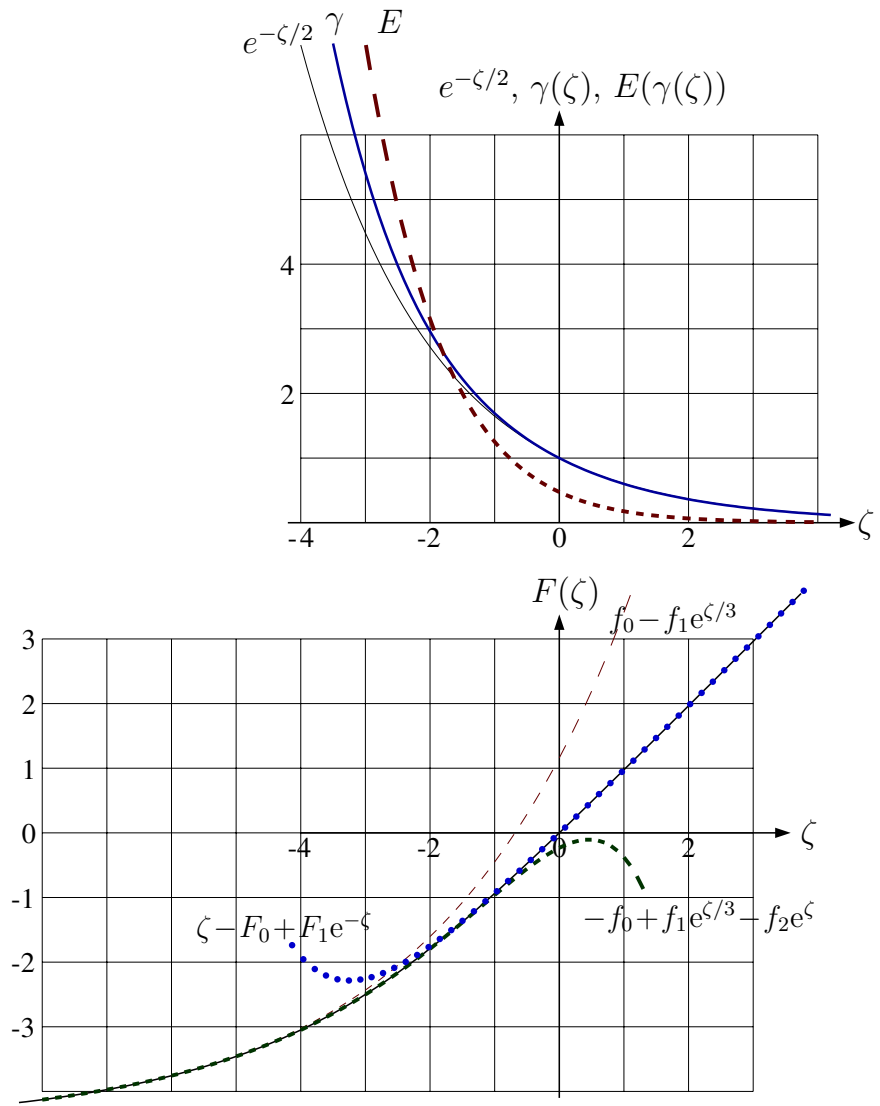

Figure 4. (a) Logamplitude $\gamma(\zeta)$ by (54), solid curve; quasi-energy $E(\gamma(\zeta))$ by (26), dashed; $\exp (-\zeta / 2)$, thin curve. (b) Normalized phase $F(\zeta)$ by (61), solid curve, and its estimates by (62), dashed and by (64), dotted.

where $t_{1}$ is time at which $\gamma=1$. Then $\gamma=\gamma(\zeta)$ satisfies the differential equation

$$
\gamma^{\prime}(\zeta)=-\frac{A(\gamma(\zeta)) k(\gamma(\zeta))}{\pi E^{\prime}(\gamma(\zeta))}, \quad \gamma(0)=1
$$

The function $\gamma(\zeta)$ is shown in figure 4 with thick solid line; it is a fixed function. For comparison, $\exp (-\zeta / 2)$ and quasi-energy $E(\gamma(\zeta))$ are shown in the same graphic.

For the evaluation of function $\gamma(\zeta)$, it is more convenient to deal with the differential equation for $G=G(\zeta)=\ln \gamma(\zeta)$;

$$
G^{\prime}=-\frac{A\left(\mathrm{e}^{G}\right) k\left(\mathrm{e}^{G}\right)}{\pi E^{\prime}\left(\mathrm{e}^{G}\right) \mathrm{e}^{G}}, \quad G(0)=0 .
$$

At $\zeta \gg 1$, the function $G$ can be approximated with

$$
G(\zeta) \approx-\frac{\zeta}{2}-\sum_{n=0}^{N-1} \frac{G_{n}}{(-\zeta)^{n}}+\mathcal{O}\left(\zeta^{-N}\right)
$$


the first coefficients in this expansion are evaluated in the same manner as in the case of the large $\gamma$ expansion of the normalized frequency $k$ :

$$
\begin{aligned}
& G_{0} \approx 0.01635242, \\
& G_{1} \approx 0.00004292, \\
& G_{2} \approx 0.0005367, \\
& G_{3} \approx 0.00208 .
\end{aligned}
$$

At $N=4, \zeta>11$, such an expansion gives at least seven significant figures.

At large negative values of $\zeta$, function $G(\zeta)$ can be approximated with

$$
G(\zeta) \approx-\frac{2}{3} \zeta-g_{0}+o(-1 / \zeta) ; \quad g_{0} \approx 0.37463601
$$

The function $o$ decays quickly; at $\zeta<-10$, this estimate gives at least eight significant figures.

From estimates (56), (58) we may expect that the function $G(\zeta)=\ln [\gamma(\zeta)]$ decreases almost linearly with time; although the tangent is little bit different for positive and negative values of the argument. At strong spiking, the decrement is slightly stronger than at weak pulsation; $G(\zeta)$ looks similar to the linear function, and $\gamma(\zeta)$ behaves like an exponential (figure 4).

It is interesting that logamplitude $\gamma$ behaves as exponential function of $\zeta$; at negative values of $\zeta$, the amplitude $X_{\max } / X_{\min }-1$ of pulsation behaves as a double-exponential function of time. This behaviour explains why the direct numerical solution of the initial system (1), (2) is so slow at the strong spiking: per each period, the conventional numerical algorithm should trace the variable which varies by many orders of magnitude. The logarithm of logamplitude, contrary, behaves very smoothly; it can be approximated with the modest function

$$
\begin{aligned}
\ln (\gamma(\zeta)) \approx G_{\text {fit }}(\zeta) & =\frac{\sqrt{(\zeta+2.1497015)^{2}+1}-7 \zeta}{12}-0.19549422 \\
& -\frac{17.8481+78.34 \zeta+11.497 \zeta^{2}+0.272 \zeta^{3}+0.0265 \zeta^{4}}{\left[(\zeta+2.6)^{2}+39.6\right]\left[(\zeta+2.025)^{2}+2.75\right] \sqrt{(\zeta-18)^{2}+375}}
\end{aligned}
$$

with four significant figures; $\left|G_{\text {fit }}(\zeta)-G(\zeta)\right|<1.4 \times 10^{-4}$. Most of the dependence is provided by the first term in the right-hand side of (59); the last fraction gives the relatively small correction, which ranges between zero and unity.

The equivalence of parameters $u$ and $v$ in the damping allows us to describe the evolution of logamplitude with fixed function of one argument; $\gamma(\zeta)$ does not depend on any parameters. This gives the description of damping of pulsation. As verification, in figure $5(a)$, the evolution of $E(\gamma(\zeta)$ ), by (26), (54), is compared to two numerical solutions of (14), for $(u=0.05, v=0)$ and for $(u=0.05, v=0)$; the $\exp \left(G_{\mathrm{fit}}(\zeta)\right)$ instead of $\gamma(\zeta)$ was used to plot the graphic. Good agreement takes place. However, the estimate does not take into account that the quasi-energy dissipates mainly in vicinity of maxima of $x$, where its derivative is high. Therefore this approximation does not reproduce the stair-like jumps of quasi-energy $E$ in the left-hand side of the graphic.

The parameter $\zeta$ by (53) determines the logamplitude $\gamma$ of pulsation, $\gamma=\gamma(\zeta)$. At $\zeta>0, \gamma(\zeta) \approx \exp (-\zeta / 2)$, reproducing the exponential decay of the weak pulsation. At $\zeta<0$ (strong pulsation), the logamplitude decays a little bit faster, $\gamma(\zeta) \sim \exp \left(-\frac{2}{3} \zeta\right)$. 


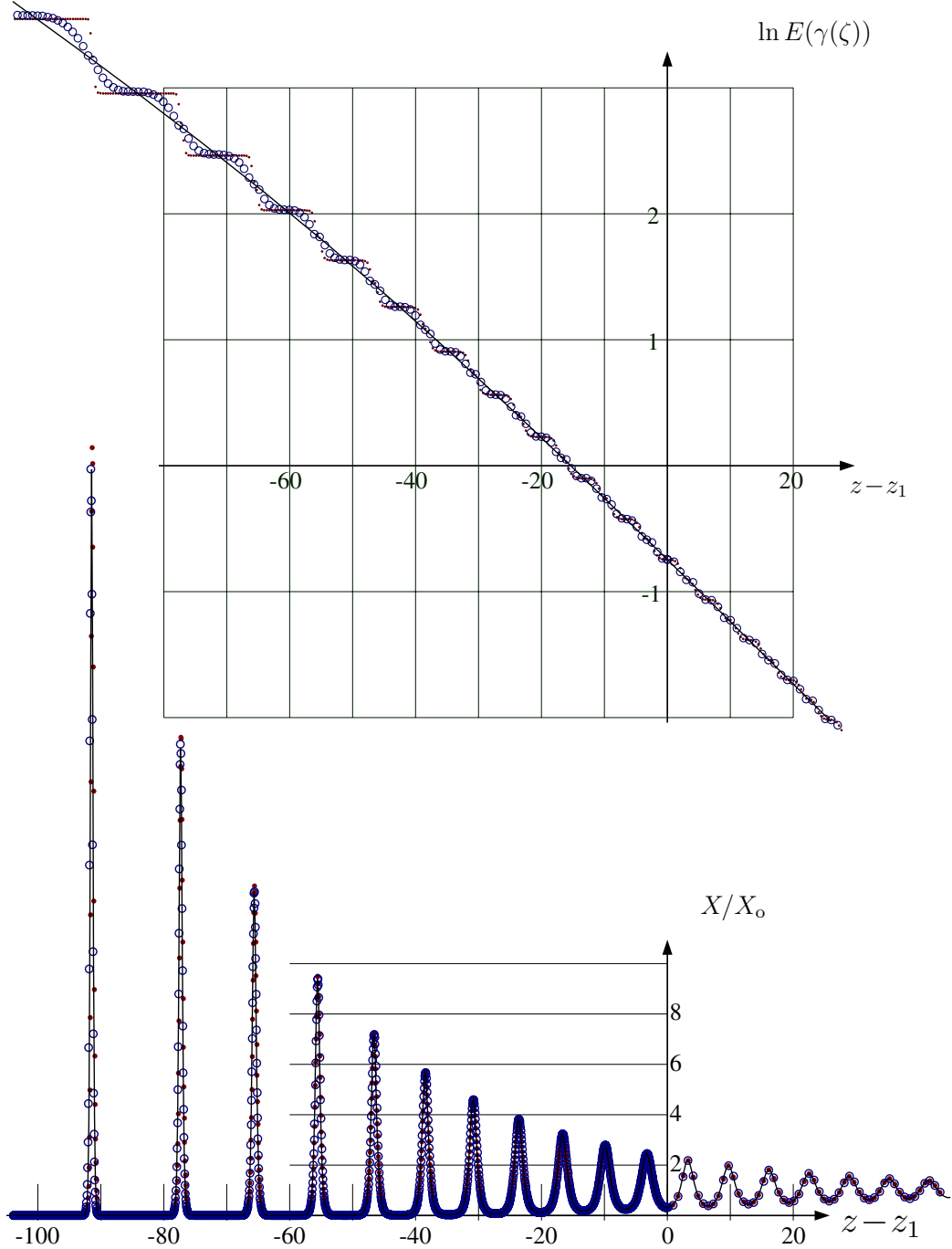

Figure 5. (a) Quasi-energy versus normalized time $z-z_{1}=\left(t-t_{1}\right) \omega$ for the case $u+v=0.05$ as $E\left(\gamma\left(\left(z-z_{1}\right) /(u+v)\right)\right)$, thin curve; quasi-energy evaluated by (17) from the numerical solution of (14) for $\{u=0.05, v=0\}$, (dots), and for $\{u=0, v=0.05\}$, (circles). (b) Normalized output power in the same cases.

\section{Phase of pulsation}

The perturbation analysis, used in the previous section for the evolution of the logamplitude $\gamma$, can be extended to take into account the phase of pulsation. The phase at the normalized time $z$ can be estimated integrating the normalized frequency $k$ of pulsation:

$$
\varphi=\int_{z_{1}}^{z} k\left(\gamma\left((u+v) z_{2}\right)\right) \mathrm{d} z_{2}=\frac{F\left((u+v)\left(z-z_{1}\right)\right)}{u+v},
$$

where $z_{1}$ is the normalized time, when $\gamma=1$, and

$$
F(\zeta)=\int_{0}^{\zeta} \mathrm{d} \tau k(\gamma(\tau))
$$


At large negative $\zeta$, the expansion may have the form

$$
F(\zeta) \approx-f_{0}+f_{1} \exp (\zeta / 3)-f_{2} \exp (\zeta)+\mathcal{O}(\exp (2 \zeta))
$$

where

$$
f_{0} \approx 4.52245451, \quad f_{1} \approx 5.683174, \quad f_{2} \approx 1.353 .
$$

At large positive $\zeta$, function $F$ can be approximated with

$$
F(\zeta) \approx-F_{0}+\zeta+F_{1} \exp (-\zeta)+\mathcal{O}\left(\exp \left(-\frac{4}{3} \zeta\right)\right)
$$

where

$$
F_{0} \approx 0.0393292835, \quad F_{1} \approx 0.039
$$

Above, we type 'may have form' instead of 'has form', because we do not yet have the formal mathematical proof; so, such expansions can be considered as our guess. The first coefficients in the expansions (62) and (64) are estimated from the numerical evaluation of (61) for large (non-physical) values of $|\zeta| \gg 1$, in the same way as in the case of functions $k$ and $A$ in the previous sections.

In the whole range, function $F$ can be approximated with

$$
\begin{array}{rl}
F_{\text {fit }}(\zeta)=-2.280 & 8919+\left(t+\sqrt{(t+4.48312523)^{2}+14.67}\right) / 2 \\
- & \frac{53856.7+16038.6 \zeta+2185.54 \zeta^{2}+134.79 \zeta^{3}+3.6687 \zeta^{4}}{\left[(\zeta+12.78)^{2}+81.611\right]\left[(\zeta+6.585)^{2}+36.7524\right] \sqrt{(\zeta+2.56)^{2}+10.36}} ;
\end{array}
$$

$\left|F_{\text {fit }}(\zeta)-F(\zeta)\right|<1.5 \times 10^{-4}$

The estimates (62) and (64) are compared to the function $F(\zeta)$ by $(61)$ in figure $4(b)$. (The graphical resolution does not allow us to see the difference between function $F$ and its fit $F_{\text {fit }}$ by (66).) These estimates reveal the basic properties of the normalized phase $F(\gamma)$. From (64) it follows that $F(-\infty)=-f_{0} \approx-4.5$. Then the phase increases, the graphic passes through the origin of coordinates, $F(0)=0$; it follows from (61). At small $\gamma \ll 1$, the phase increases linearly, which corresponds to the linear oscillator with frequency not dependent on the amplitude $[1,2]$.

The minimal value of the normalized phase $-f_{0} \approx-4.52245451$ indicates that the maximal number of strong peaks is determined by the sum $u+v$. Even at high initial amplitude of pulsation, within

$$
N_{\max }=\frac{f_{0}}{2 \pi(u+v)}
$$

pulses, the logamplitude of pulsation becomes smaller than unity. In particular, for the case $u+v=0.05$ shown in the figure 5, there may exist no more than 14 pulses with logamplitude larger than unity; 11 of them are within the plot range.

The approximation (62) allows us to estimate the maximal number of strong spikes. At weak damping $(u+v \ll 1)$, independently on the initial conditions, the number of strong pulses does not exceed $N_{\max }$ by (67).

\section{Approximation of the output power}

The knowledge of behaviour of logamplitude $\gamma=\gamma(\zeta)$ and phase $\varphi=F(\zeta) /(u+v)$ allows us to approximate the number of photons $X$ and the output power (7) with simple functions. Generalizing the approximations $x_{j}$ by (36)-(38), consider

$$
\tilde{x}_{j}(z)=x_{j}\left(\gamma(\zeta), \frac{F(\zeta)}{u+v}+\psi\right), \quad \zeta=(u+v)\left(z-z_{1}\right)
$$


where $\psi$ is phase of pulsation at the moment $t_{1}=z_{1} / w$, when the logamplitude $\gamma=1$. The fit (68) approximates $x$ as a function of $K, W, U, V$ through $u$ and $v$; this fit can be interpreted as an approximation of a function of five parameters $\left(t-t_{1}, \psi, K, U, V\right)$, valid for $u+v \ll 1$. The number of photons in the cavity can be estimated as $\tilde{X}_{2}(t)=X_{\mathrm{o}} \exp \left(\tilde{x}_{2}(w t)\right)$. At $\psi=0, u+v=0.05$, this approximation is plotted in figure $5(b)$ with thin curve; the approximations $\exp \left(G_{\mathrm{fit}}(\zeta)\right)$ and $F_{\mathrm{fit}}(\zeta)$ were used instead of $\gamma(\zeta)$ and $F(\zeta)$. For comparison, in the same figure, the numerical solutions of (14) are shown for the cases $u=0.05, v=0$ (dots) and $u=0, v=0.05$ (circles). Both numerical curves are close to the approximation with (68). The small difference is seen at the left-hand side of figure 5, when the oscillator loses most of its energy during a small fraction of quasi-period.

The left-hand side of figure 5 is at the edge of the physical range of values of the dimension-less time $z$. At $z=-100$, we have $\zeta=-100(u+v)=-5$ and $G(-5) \approx 3$; $\gamma(-5) \approx e^{3} \approx 20$ means that the peak number of photons $X_{\max } \approx e^{40} X_{\min } \approx 3 \times 10^{17} X_{\min }$. This should correspond to enormous energy, stored in the photons inside the cavity; therefore we expect, our approximation works for all realizations of the oscillator Toda as a self-pulsing laser, while $u+v<0.05$.

In our approximation, the decay of the pulsation is determined by the sum $u+v$ and is not sensitive to the difference $u-v$. The two functions (logamplitude and phase) of variable $\zeta=w t /(u+v)$ describe the decay of pulsation. The sum $u+v$ determines also the maximal number $N_{\max }$ of strong spikes by (67). The logarithm of the logamplitude is approximated with $G_{\text {fit }}$ by (59) and the phase is approximated with $F_{\text {fit }}$ by (66). Then, the number of photons $X$ (and therefore, the output power) can be approximated with (68). This approximation is valid at $u+v<0.05$.

\section{Discrete photons and the physical interpretation}

Initial classical equations (1) do not take into account the discrete character of photons. Physically, the self-pulsation begins with photons of the spontaneous emission; the classical equations become valid as soon as these photons get amplified. This section analyses the limit on the amplitude of pulsation which arises from the discrete character of photons, and suggests the scenario of development of self-pulsation.

Assume that the pulsation begins when the medium is pumped just to reach the threshold of the laser action. This position can be interpreted as minimum of the output power. Assume, there is of order of one photon in the cavity, $X_{\min }=1$; this is approximation of the quantum process in classical terms. The initial value of $x$ is expressed with logarithm of $X$; therefore, it will not affect much the evolution, if we take several photons in the initial condition. Then, at the maximal logamplitude of pulsation, $\gamma+\delta=\ln X_{\mathrm{o}}$, and (25) gives

$$
2 \gamma=\ln \left(X_{\mathrm{o}}\right)+\ln (2 \gamma)+\mathcal{O}(\exp (-2 \gamma))
$$

The maximal value of logamplitude

$$
\gamma_{\max } \approx \frac{1}{2} \ln \left(X_{\mathrm{o}}\right)+\frac{1}{2} \ln \left(\ln \left(X_{\mathrm{o}}\right)\right)=\frac{1}{2} \ln \left(X_{\mathrm{o}} \ln \left(X_{\mathrm{o}}\right)\right)
$$

The maximal number of photons can be estimated as

$X_{\mathrm{MAX}}=X_{\mathrm{o}} \exp \left(\gamma_{\max }-\delta_{\max }\right) \approx 2 \gamma X_{\mathrm{o}} \approx X_{\mathrm{o}}\left[\ln \left(X_{\mathrm{o}}\right)+\ln \left(\ln \left(X_{\mathrm{o}}\right)\right)\right] \approx X_{\mathrm{o}} \ln \left(X_{\mathrm{o}}\right)$.

The above estimates lead to the simplified scenario of development of self-pulsation. As the pump is switched on, there are almost no photons in the cavity, until the number of excitation of medium reaches its steady-state value $Y_{\mathrm{o}}$; this is threshold of the laser action. If we neglect the depletion of excitation due to the spontaneous emission, then this process takes 
time $t_{0}=Y_{\mathrm{o}} / W$. Then the photons of spontaneous emission get amplified, and their number can be interpreted as a continuous commutative variable.

The initial value of the normalized dimension-less time $\zeta$ can be estimated from the asymptotic expansion (58); this gives the estimate

$$
-\zeta_{\mathrm{o}} \approx \frac{3}{2}\left[g_{0}+\ln \left(\gamma_{\max }\right)\right] \approx \frac{3}{2}\left[g_{0}+\frac{1}{2} \ln \left(X_{\mathrm{o}} \ln \left(X_{\mathrm{o}}\right)\right)\right]
$$

The logamplitude of self-pulsation decays almost exponentially, the laser produces of order of $N_{\max }$ strong pulses during time

$$
t_{1}=\frac{-\zeta_{0}}{(u+v) w} .
$$

Then logamplitude becomes of order of unity and smaller; the rate of decay reduces from $\frac{2}{3}(u+v) w$ to $\frac{1}{2}(u+v) w$, and the pulsation becomes decaying sinusoidal, and the analytical description by $[1,2]$ becomes valid; after several times $(u+v)^{-1} / w$, the pulsation disappears at the background of the statistical noise. During the quasi-periodic pulsation, the output power can be approximated with fit $x_{2}$ by (38), (68).

\section{Comparison with experiments}

This section describes our attempt to realize the oscillator Toda at the optical bench. Although experimental measurements of various self-pulsing lasers have been frequently reported, not so many authors compare the measured and calculated pulse traces [19, 20, 23, 24]. In figure 6, we compare our original oscillograms to the model considered above.

Our laser is a $1 \mathrm{~mm}$ thick, $10 \%$ at ytterbium-doped, $\mathrm{Y}_{3} \mathrm{Al}_{5} \mathrm{O}_{12}$ ceramic disc obtained from Konoshima Chemical Corp [33]. A multilayer coating, anti-reflective at the pump wavelength $\lambda_{\mathrm{p}}=940 \mathrm{~nm}$, and highly reflective for the signal wavelengths $\lambda_{\mathrm{s}}=1030 \mathrm{~nm}$, is deposited on one face. The output coupler consists of a multilayer coating with $\theta=10 \%$ transmission factor at the signal wavelength. Laser operation of the device was performed by axial pumping through the rear mirror with a semiconductor laser diode (LIMO Corp.), delivering up to $26 \mathrm{~W}$ at $\lambda_{\mathrm{p}}=940 \mathrm{~nm}$. The pump was delivered with a $1 \mathrm{~m}$ long, $200 \mu \mathrm{m}$ diameter fibre with a numerical aperture $\mathrm{NA}=0.22$. A pair of aspherical lenses with focal length $f=8 \mathrm{~mm}$ and NA $=0.5$ was used to concentrate the pump light on the microchip with a transmission efficiency of $96 \%$. The spot size was measured by the knife-edge method and found to contain $90 \%$ of the total power in a circle of radius about $80 \mu \mathrm{m}$. The pump absorption efficiency was measured to be around $75 \%$. The output power as a function of time measured with the optical detector EG\&G:G8194-32 and the Tektronix TDS 3052B oscilloscope. We calibrated the detector with 'LaserMate' powermeter from Coherent.

Pulsed pumping was used to access the transient regime of this laser. Input pump power was reduced to $0.5 \mathrm{~W}$ in order to mitigate the thermal effects; this corresponds to absorbed power $P_{\mathrm{p}}=375 \mathrm{~mW}$. The pulse duration was $2 \mathrm{~ms}$, with a $10 \mu \mathrm{s}$ rising front. We assume the following values of parameters: $\sigma=2 \times 10^{-20} \mathrm{~cm}^{2}, \tau=0.97 \mathrm{~ms}, n=1.8$. Using definitions (3)-(6), we evaluate $K=5.3 \times 10^{-6} \mathrm{~Hz}, U=5.3 \times 10^{9} \mathrm{~Hz}, V=1030 \mathrm{~Hz}, W=$ $1.77 \times 10^{15} \mathrm{~Hz}$. With equations (8)-(6), we estimate $X_{\mathrm{o}}=1.4 \times 10^{8}, Y_{\mathrm{o}}=10^{15}, \Gamma=1775 \mathrm{~Hz}$ and $w=\sqrt{K W-U V}=2 \times 10^{6} \mathrm{~Hz} \approx \Omega$. Note that $\Omega \gg \Gamma$; this means negligible damping: $u \ll 1, v \ll 1$.

We plot two examples of the oscillograms in figure 6 . The period of small pulsation is expected to be $T_{\mathrm{o}}=2 \pi / \Omega=3.16 \times 10^{-6} \mathrm{~s}$, which is slightly larger than the quasi-period of pulsation at the right hand side of figure 6 . The strong spiking is seen at the left-hand side of figure. The maximum number of photons $X_{\max }$ is about five times larger than the average 


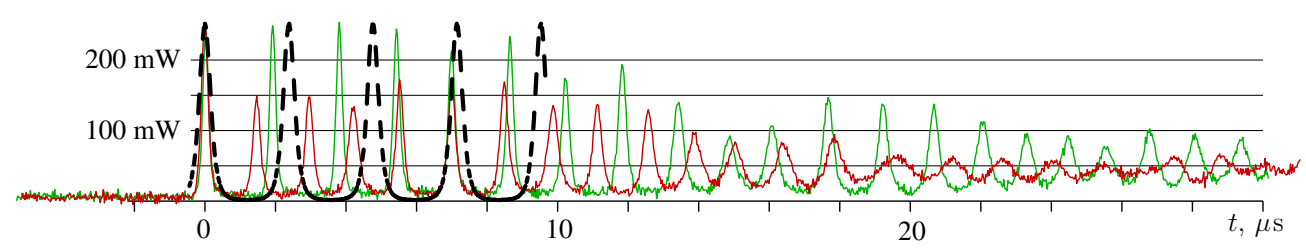

Figure 6. Output power versus time since the first maximum: two experimental realizations of the self-pulsation (thin curves) and the approximation using equation (7) (dashed curve).

value $X_{\mathrm{o}}$, i.e. $X_{\max } / X_{\mathrm{o}} \approx 5$. From equation (70), we estimate the initial value of logamplitude $\gamma \approx 2.5$. Using equation (27), we estimate the period $T \approx 2.6 \mu \mathrm{s}$. Both the period and the pulse width are qualitatively reproduced by our theoretical model.

We also compared the absolute output power to the predictions of our model. In the transient regime, the average output power is about one third of that predicted by (7). Therefore, we scale down the theoretical estimate with factor $1 / 3$; this scaled estimate is shown in figure 6 with dashed curve. This scaling means that our model overestimates the output power at the transient regime.

We cannot predict well the moment of the beginning of pulsation, this moment fluctuates from the estimate $T_{1}=\frac{Y_{0}}{W}=\frac{U}{K W}$ of time necessary to deliver the active medium the steadystate number $Y_{\mathrm{o}}$ of excitations. Therefore, we displace the time scale for the two realizations to show them at the same graphic in figure 6.

The relaxation to the steady state is faster than what our model predicts; at $u+v \approx 10^{-4}$, no significant decay would be seen in figure 6 . The oscillograms look like a beat of pulsation of several competing modes [23, 24].

The necessity to scale the ordinate axis in figure 6 and to displace the abscissas axis and the visible decay of the pulsations means that we do not fully reproduce the equations (1) at the optical bench; as [19], we got only qualitative agreement. Various effects may affect the system: the re-absorption at the signal wavelength $[19,16]$, the modal structure of the signal $[23,24]$, non-uniform distribution of pump and signal, the thermal lensing and the spatial hole burning [34, 35]. A laser, which follows the scenario for the oscillator Toda, is still left to be achieved.

\section{Conclusions}

The simple model of self-pulsation in lasers is based on equations (1), (2). The model includes four parameters $K, U, V, W$, which determine the rate of conversion of energy of excitation of the active medium to the lasing photons, rate of escape of photons from the cavity, rate of relaxation of the active medium and rate of its pumping. In such a system, the pulsation with frequency scale $w=\sqrt{K W-U V}$ may take place. This model is equivalent to the oscillator Toda [3] with dimensionless damping parameters $u=w / U$ and $v=V / w$ (14). The spiking is possible at low damping, $0<u \ll 1$ and $0<v \ll 1$; then, the damping term can be considered as perturbation. The loss of quasi-energy during the half-period of pulsation determines the decay. The state of the equivalent oscillator is characterized by the logamplitude $\gamma$ and phase $\varphi$, which are approximated through elementary functions (59) and (66) of normalized dimensionless time parameter $\zeta=(u+v)\left(t-t_{1}\right) w$.

The approximation (66) indicates the maximal number of strong spikes. Even at high initial amplitude of pulsation, within $N_{\max } \approx 0.72 /(u+v)$ spikes, the logamplitude of pulsation 
becomes smaller than unity. At strong pulsation, the logamplitude $\gamma$ decays with decrement $\frac{2}{3}(u+v) w$. Then, the decrement becomes $\frac{1}{2}(u+v) w$, self-pulsation becomes linear and can be described with the linearized equations $[1,2]$. The discrete (quantum) character of photons limits the range of validity of the model for very strong pulsation; the maximal value of logamplitude $\gamma_{\max } \approx \frac{1}{2} \ln \left(X_{\mathrm{o}} \ln \left(X_{\mathrm{o}}\right)\right)$. The peak number $X_{\max }$ of photons in the cavity does not exceed value $X_{\mathrm{MAX}} \approx X_{\mathrm{o}} \ln \left(X_{\mathrm{o}}\right)$. The first spike comes with delay of order of $\sqrt{\gamma_{\max }} / w$ after reaching the threshold of the laser action.

The precision of the estimates above for equations (1), (2) exceeds the precision of the realizations of oscillator Toda as a pulsed laser. An accurate experimental realization of the oscillator Toda (14) remains a challenging task. Also, the model can be updated to describe better the typical experimental conditions. The consideration of logarithms of the numbers of photons in various modes might give the simple description of the competition between longitudinal modes $[23,24]$. The role of spatial distribution of pump in the active medium, and the gradual pumping up of the lateral region of the sample, and thermal drift of the size of the signal mode in the transient regime could be considered in the similar way, treating the initial parameters in equation (1) as slow functions of time. In such a way, the approximations (34), (52) could also be a tool for more complicated models.

\section{Acknowledgments}

Thanks to J Dong for the discussions. This work was supported by the 21 st Century COE program of the Ministry of Education, Science and Culture of Japan.

\section{References}

[1] Siegman A 1986 Lasers (Sausalito CA: University Science Books)

[2] Koechner W 1999 Solid-State Laser Engineering (New York: Springer)

[3] Oppo G L and Politi A 1985 Toda potential in laser equations Z. Phys. B 59 111-5

[4] Kouznetsov D, Bisson J-F, Takaichi K and Ueda K 2005 High-power single-mode solid-state laser with short wide unstable cavity J. Opt. Soc. Am. B 22 1605-19

[5] Kouznetsov D, Bisson J-F, Dong J and Ueda K 2006 Surface loss limit the power scaling of thin-disk laser J. Opt. Soc. Am. B 23 1074-82

[6] Basov N G 1968 O1 dynamics of injection lasers IEEE J. Quantum Electron. 4 855-64

[7] Dixon R W and Joyce W B 1979 A possible model for sustained oscillations (Pulsations) in (Al, Ga)As doubleheterostructure lasers IEEE J. Quantum Electron. 15 470-4

[8] Ueno M and Lang R 1985 Conditions for self-sustained pulsation and bistability in semiconductor lasers J. Appl. Phys. 58 1689-92

[9] Yamada M 1993 A theoretical analysis of self-pulsation phenomena in narrow-strip semiconductor lasers IEEE J. Quantum Electron. 29 1330-6

[10] Mirasso C R, van Tartwijk G H M, Hernández-García E, Lenstra D, Lynch S, Landais P, Phelan P, O’Gorman J, San Miguel M and Eläßer W 1999 Self-pulsating semiconductor lasers: Theory and experiment IEEE J. Quantum Electron. 35 764-70

[11] Smith P W 1967 The self-pulsing laser oscillator IEEE J. Quantum Electron. 3 627-35

[12] Li J, Ueda K I, Musha M, Shirakawa A and Zhong L 2006 Self-pulsing dynamics of ytterbium-doped fiber laser with pump-bypassed cavity Appl. Phys. B 85 565-9

[13] Danielmeyer H G 1970 Low-frequency dynamics of homogeneous four-level cw lasers J. Appl. Phys. 41 4014-18

[14] Koechner W 1972 Output fluctuations of CW-pumped Nd:YAG lasers IEEE J. Quantum Electron. 8 656-61

[15] Fan T Y and Byer R L 1987 Modeling and CW operation on a quasi-three level $946 \mathrm{~nm}$ Nd:YAG laser IEEE J. Quantum Electron. 23 605-12

[16] Risk W P 1988 Modeling of longitudinally pumped solid-state lasers exhibiting reabsorption losses J. Opt. Soc. Am. B 5 1412-23 
[17] Marcuse D 1993 Pulsing behavior of a three-level laser with saturable absorber IEEE J. Quantum Electron. 29 2390-6

[18] Shahruz S M and Mahavaraha T A 2000 A system theoretic approach to the self-pulsation of passively Qswitched lasers IEEE Trans. Control Syst. Technol. 8 358-65

[19] Tronciu V Z, Yamada M, Ohno T, Ito S, Kawakami T and Taneya M 2003 Self-pulsation in an InGaN lasertheory and experiment IEEE J. Quantum Electron. 39 1509-14

[20] Tang D Y, Ng S P, Qin L J and Meng X L 2003 Deterministic chaos in a diode-pumped Nd:YAG laser passively $\mathrm{Q}$ switched by a $\mathrm{Cr}^{4+}$ : YAG crystal Opt. Express 28 325-7

[21] Jackson S D 2002 Direct evidence of the laser reabsorption as initial cause for self-pulsing in three-level fibre lasers Electron. Lett. 38 1640-2

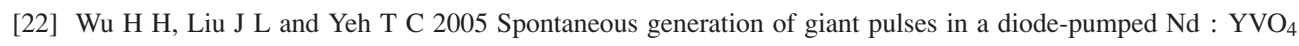
laser Opt. Express 13 3174-8

[23] Dong J, Shirakawa A and Ueda K 2005 Numerical simulation of a diode-laser-pumped self-Q-switched Cr,Yb:YAG microchip laser Opt. Rev. 12 170-8

[24] Dong J and Ueda K 2005 Longitudinal mode competition induces instabilities of $\mathrm{Cr}^{4+}, \mathrm{Nd}^{3+}: \mathrm{Y}_{3} \mathrm{Al}_{5} \mathrm{O}_{12} \mathrm{self}$ Q-switched two-mode laser Appl. Phys. Lett. 87151102

[25] Tsang Y H, King T A, Ko D K and Lee J 2006 Output dynamics and stabilization of a multi-mode double-clad Yb-doped silica fibre laser Opt. Commun. $259236-41$

[26] Bornholdt C, Sartorius B, Schelhase S, Möhrle M and Bauer S 2000 Self-pulsating DFB laser for all-optical clock recovery at $40 \mathrm{Gbit} / \mathrm{s}$ Electron. Lett. 36 327-8

[27] Renaudier J et al 200545 GHz self-pulsation with narrow linewidth in quantum dot Fabry-Perot semiconductor lasers at $1.5 \mu \mathrm{m}$ Electron. Lett. 41 1007-8

[28] Matorin I I, Pikolskii A S and Khanin Ya I 1984 Multistability and autostochasticity in a laser with a delayedresponse active medium subjected to periodic loss modulation Sov. J. Quantum Electron. 14 1401-5

[29] Ratner A M 1972 Spectral, Spatial and Temporal Properties of Lasers (New York: Plenum)

[30] Wázquez J L 2003 The nonlinearly damped oscillator ESAIM-Control Opt. Calculus Variations 9 231-46

[31] Lim C W and Lai S K 2006 Accurate higher-order analytical approximate solutions to nonconservative nonlinear oscillators and application to van der Pol damped oscillators Int. J. Mech. Sci. 48 483-92

[32] Mickens R E 2006 Investigation of the properties of the period for the nonlinear oscillator $\ddot{x}+\left(1+\dot{x}^{2}\right) x=0 \mathrm{~J}$. Sound Vib. 292 1031-5

[33] http://www.konoshima.co.jp

[34] Zayhowski J J 1999 Microchip lasers Opt. Mater. 11 255-7

[35] Casperson L W 1980 Laser power calculation: sources of error Appl. Opt. 19 422-34 